\title{
Signal Transduction Events in Stimulated Rat Neutrophils: Effects of Adenine Nucleotides ${ }^{1}$
}

\author{
Peter A. Ward, ${ }^{2}$ Thomas W. Cunningham, and Kent J. Johnson \\ Department of Pathology. The University of Michigan Medical School, 1301 Catherine Street, \\ Ann Arbor, Michigan 48109
}

\begin{abstract}
When rat neutrophils were stimulated with chemotactic peptide $(N$ formyl-Met-Leu-Phe; fMLP), phorbol myristate acetate (PMA), or immune complexes in the presence of homologous platelets, $\mathrm{O}_{2}$ responses were enhanced. Secretion products of thrombin-stimulated platelets as well as ATP. ATP $\gamma S$, or ADP enhanced $\mathrm{O} \dot{2}$ responses of fMLP-stimulated neutrophils, although these nucleotides did not, by themselves, initiate an $\mathrm{O} \doteqdot$ response. Calcium transients were measured in neutrophils which were stimulated with fMLP under a variety of conditions ( \pm ATP $\gamma \mathrm{S}, \pm$ cytochalasin $B$ ) which enhance $\mathrm{O} \dot{2}$ responses. Neutrophils incubated with ATP $\gamma S$ alone developed brief calcium transients similar to those produced by fMLP. As compared to changes in intracellular calcium in fMLP-stimulated neutrophils, the copresence of ATP $\gamma \mathrm{S}$ with fMLP did not cause a statistically significant difference in the calcium transients, even though $\mathrm{O} \frac{-}{2}$ production was enhanced. In contrast, in the presence of cytochalasin $B$, the addition of $\mathrm{ATP} \gamma \mathrm{S}$ to fMLP-stimulated neutrophils produced greatly sustained and protracted elevations in intracellular calcium, correlating with further enhancement of $\mathrm{O} \frac{1}{2}$ responses. In fMLP-stimulated neutrophils the latter phases of the calcium transients appeared to be dependent in part on the availability of extracellular calcium. These data suggest that ATP $\gamma S$-induced enhancement of $\mathrm{O} \div$ responses in fMLP-stimulated rat neutrophils may be related to two mechanisms, one being independent of fMLP-induced intracellular calcium transients and the other, which is related to the presence of cytochalasin $\mathbf{B}$, being linked to sustained elevations in intracellular calcium associated with mobilization of extracellular calcium. (C) 1989 Academic Press. Inc.
\end{abstract}

\section{INTRODUCTION}

There is accumulating evidence that important interactions occur between platelets and neutrophils, the outcome of which may be intensification of the inflammatory response. We have recently described an interaction between human platelets and neutrophils, resulting in amplified oxygen radical generation by neutrophils (1). This interaction is largely independent of the nature of the neutrophil agonist (e.g., similar effects are found with chemotactic formyl peptide, phorbol ester, immune complexes) and can be reproduced by the substitution of intact platelets with lysates of platelets or thrombin-induced secretion products from platelets. The active principle in the human platelet has been recently identified as ATP/ADP (1).

\footnotetext{
${ }^{1}$ Some of these data were presented in honor of Professor Elmer L. Becker at a symposium held at the University of Connecticut Health Center, Farmington, CT, on April 4, 1988. The article is dedicated to Dr. Becker who served as an important preceptor for some of Dr. Ward's early scientific training.

${ }^{2}$ To whom correspondence should be addressed.
} 
Enhancement of superoxide responses of fMLP-stimulated human neutrophils can be obtained by addition of ATP or ADP or their hydrolytically stable analogs (2). The adenine nucleotides do not themselves initiate in the neutrophil an oxygen radical response. However, nucleotides appear to be able to modify the neutrophil in a manner suggesting that it has been "primed" for an enhanced superoxide response. Manipulation of neutrophils with inhibitors of $S$-adenosyl homocysteine hydrolase or adenosine deaminase causes suppressive effects on superoxide responses of stimulated neutrophils (2), suggesting that metabolism of the adenine nucleotide to a product beyond adenosine does not account for the observed effects of the nucleotides on superoxide responses.

The current studies were initiated in rat neutrophils in order to further define the signal-transduction mechanisms that may be responsible for the enhanced functional responses of neutrophils by adenine nucleotides. The focus of these studies was whether or not the adenine nucleotide-induced enhancement of superoxide responses could be related to changes in intracellular calcium.

\section{MATERIALS AND METHODS}

\section{Chemicals and Reagents}

All materials were purchased from Sigma Co. (St. Louis, MO) except as indicated.

\section{Preparation of Rat Neutrophils and Platelets}

Young adult male Long-Evans rats (Charles River Laboratory, Charles River, MA) were used. Neutrophils were obtained from glycogen-induced peritoneal exudates $4 \mathrm{hr}$ after intraperitoneal injection of $0.1 \%$ glycogen (3). When present, erythrocytes were removed by hypotonic lysis with $0.15 \mathrm{M}$ ammonium chloride. Cells were suspended in Hanks' balanced salt solution (HBSS) and consisted of $>96 \%$ ncutrophils as assessed morphologically. No platelets were detected in these cell suspensions.

Platelet preparation was achieved by obtaining blood from the inferior vena cava of anesthetized rats. The blood was drawn into acid-citrate-dextrose, maintaining a blood:citrate solution ratio of 10:1. The blood suspension was subjected to centrifugation at $250 \mathrm{~g}$ for $15 \mathrm{~min}$ at room temperature. The overlying plateletrich plasma was obtained by aspiration, diluted 1:1 with HBSS (without addition of calcium and $\mathrm{Mg}^{2+}$ ) in the presence of $0.035 \%$ EDTA, and then subjected to centrifugation ( $900 \mathrm{~g}$ for $12 \mathrm{~min}$ ) at room temperature. The platelet-rich suspension was aspirated, washed once in the same salt solution, and the platelets were then suspended in HBSS to which calcium chloride and magnesium sulfate were subsequently added. The yield from $10 \mathrm{ml}$ platelet-rich plasma was approximately $10^{8}$ platelets $/ \mathrm{ml}$. The platelets showed very little contamination with neutrophils $(<100 / \mathrm{ml})$. Contaminating monocytes and lymphocytes were also rare.

\section{Preparation of Immune Complexes}

Insoluble immune complexes were prepared with polyclonal rabbit IgG anti- 
body to bovine serum albumin (BSA) in a weight ratio of 5:1 (antibody:antigen) as previously described (3).

\section{Measurement of $\mathrm{O}_{2}$ Responses of Neutrophils}

Rat neutrophils $\left(2 \times 10^{6}\right)$ suspended in HBSS with calcium and $\mathrm{Mg}^{2+}$ were incubated with the appropriate agonist at $37^{\circ} \mathrm{C}$ for a period of $30 \mathrm{~min}$ with constant agitation. The final reaction volume was $1.5 \mathrm{ml}$. Production of $\mathrm{O}_{2}^{-}$was calculated based on the ferricytochrome $c$ reduction which could be inhibited by the presence of superoxide dismutase (3). The amount of immune complex employed is described in the relevant experiment. Unless otherwise indicated, immune complexes and the chemotactic peptide, fMLP, were used in the presence of cytochalasin $\mathrm{B}(5 \mu \mathrm{g} / \mathrm{ml})$ which is known to amplify $\mathrm{O} \dot{2}$ responses of rat neutrophils (3). Phorbol myristate acetate (PMA; LC Services Corp., Woburn, MA) was dissolved in dimethyl sulfoxide (DMSO) at a concentration of $1 \mathrm{mg} / \mathrm{ml}$ and then diluted in HBSS to achieve the desired final concentration. Zymosan particles were first boiled in distilled water, washed in HBSS, and then added to fresh rat serum $\left(10 \mathrm{mg}\right.$ zymosan particles $/ \mathrm{ml}$ ) and incubated with constant agitation at $37^{\circ} \mathrm{C}$ for $1 / 4 \mathrm{hr}$. The mixture was then diluted 10 -fold with HBSS and the particles were washed twice before final suspension after centrifugation in HBSS. When fMLP was used, it was first dissolved in DMSO at a stock concentration of $10^{-2} M$ and then diluted in HBSS.

\section{Measurement of Intracellular Calcium}

Neutrophils were loaded with Fura 2/AM (acetoxymethyl ester of Fura 2; Calbiochem, La Jolla, CA) by incubation at $37^{\circ} \mathrm{C}$ in salt solution with Hepes buffer (150 mM NaCl, $5.6 \mathrm{~m} M \mathrm{KCl}, 2 \mathrm{mM} \mathrm{CaCl}{ }_{2}, 1.0 \mathrm{~m} M \mathrm{MgSO}_{2}, 10 \mathrm{~m} M$ glucose, and $10 \mathrm{~m} M$ Hepes, pH 7.3). Fura $2 / \mathrm{AM}(20 \mu M)$ was added to $5 \times 10^{7}$ cells per ml, with incubation at $37^{\circ} \mathrm{C}$. The cells were then filtered through gauze to remove small cellular aggregates, subjected to centrifugation, and resuspended to $5 \times 10^{6}$ cells

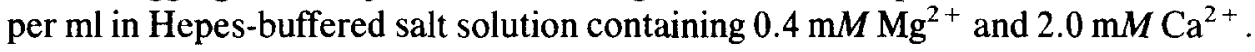
From these stock solutions, which were maintained at $4^{\circ} \mathrm{C}, 1.0-\mathrm{ml}$ aliquots were withdrawn, warmed to $37^{\circ} \mathrm{C}$, and then placed in a $1-\mathrm{cm}$ quartz cuvette to which were added various reagents. Fluorescence was monitored over a period of $4 \mathrm{~min}$ (following addition of $25 \mathrm{uM}$ adenine compounds in the presence or absence of fMLP) in a Perkin Elmer LS-5B luminescence spectrophotometer using an excitation wave length of $342 \mathrm{~nm}$ and an emission wave length of $500 \mathrm{~nm}$. For experiments in which the requirement for extracellular calcium was explored, $1.8 \mathrm{mM}$ $\mathrm{Ca}^{2+}$ was added to the neutrophil suspension at the time of addition of ATP $\gamma \mathrm{S}$ and/or fMLP. After the measurements were obtained, each sample was calibrated by disrupting the cells with $0.2 \%$ Triton $\mathrm{X}-100$ to obtain a measure of the maximum fluorescence $\left(F_{\max }\right)$ achievable with calcium. EGTA $(10 \mathrm{mM}, \mathrm{pH} 10.0)$ was subsequently added to determine the minimal fluorescence $\left(F_{\min }\right)$. Levels of intracellular $\mathrm{Ca}^{2+}$ were calculated using the equation:

$$
\left[\mathrm{Ca}^{2+}\right]_{\mathrm{i}}=K_{d} \frac{F-F_{\min }}{F_{\max }-F},
$$




\section{Statistics}

Values were expressed as the mean \pm standard error of the mean (SEM). Statistical significance was judged by one-way analysis of variance and the Student-Newman-Keuls test. A $P$ value of $<0.05$ was accepted as significant.

\section{RESULTS}

\section{Enhancement of $\mathrm{O} \div$ Responses of Stimulated Neutrophils by Platelets and Platelet Products}

Rat peritoneal neutrophils were stimulated with different agonists in the presence or absence of intact platclets or platelet secretion products. Agonists included 100 ng PMA, immune complexes containing $100 \mu \mathrm{g}$ IgG rabbit polyclonal antibody to bovine serum albumin, and a dose range of the chemotactic peptide fMLP. When immune complexes and fMLP were used as the agonists, the neutrophils were suspended in the presence of cytochalasin B $(2.5 \mu \mathrm{g} / \mathrm{ml})$. The experiments were carried out using $2 \times 10^{6}$ rat neutrophils in the presence or absence of $5 \times 10^{7}$ rat platelets or platelet secretion products which were obtained when 1.0 unit thrombin was incubated with $5 \times 10^{7}$ platelets at $37^{\circ} \mathrm{C}$ for $10 \mathrm{~min}$, followed by centrifugal removal of the platelets. The results are shown in Table 1. When agonist was omitted from the neutrophil suspension, platelets did not enhance the basal production of $\mathrm{O} \div$ (Experiment $\mathrm{A}$ ). With PMA, the presence of platelets led to a $32.2 \%$ increase $(P<0.004)$ in the amount of $\mathrm{O} \dot{2}$ generated. Similarly, in the presence of intact platelets, the $\mathrm{O} \dot{2}$ response of immune complex-

TABLE 1

Ability of Intact Platelets and Platelet Supernatant Fluids to Enhance $\mathrm{O}_{2}^{-}$ Responses of Stimulated Rai Neutrophils

\begin{tabular}{|c|c|c|c|}
\hline Experiment $^{a}$ & Material added to neutrophils ${ }^{b}$ & $\begin{array}{l}\mathrm{O}_{2}^{-} \text {response } \\
(\mathrm{nmol} / 1 / 2 \mathrm{hr})\end{array}$ & Significance ${ }^{c}$ \\
\hline & & $\bar{x} \pm \mathrm{SEM}$ & $P$ values \\
\hline \multirow[t]{6}{*}{ A } & None & $0.660 \pm 0.161$ & \\
\hline & None + platelets & $0.554 \pm 0.142$ & NS \\
\hline & PMA & $11.8 \pm 0.600$ & \\
\hline & PMA + platelets & $15.6 \pm 0.541$ & $<0.004$ \\
\hline & Immune complexes & $11.2 \pm 0.728$ & \\
\hline & Immune complexes + platelets & $14.3 \pm 0.760$ & $<0.02$ \\
\hline \multirow[t]{2}{*}{ B } & fMLP & $7.41 \pm 2.90$ & \\
\hline & fMLP + platelets & $12.39 \pm 3.91$ & $<0.04$ \\
\hline \multirow[t]{2}{*}{$\mathrm{C}$} & fMLP & $3.59 \pm 0.251$ & \\
\hline & fMLP + platelet supernatant fluid ${ }^{d}$ & $7.22 \pm 0.220$ & $<0.002$ \\
\hline
\end{tabular}

${ }^{a}$ In each experiment $(A-C)$, four separate and independent series of determinations were made. In all cases cytochalasin $(2.5 \mu \mathrm{g} / \mathrm{ml})$ was present in the neutrophil suspension.

${ }^{b}$ Reaction conditions: neutrophils, $2 \times 10^{6}$; platelets, $5 \times 10^{7}$; PMA, $100 \mathrm{ng}$; immune complexes containing $100 \mu \mathrm{g}$ antibody; fMLP, $10^{-6} \mathrm{M}$.

${ }^{c}$ In each case the second set of values was compared to the first set of values.

${ }^{d}$ Supernatant fluids from $5 \times 10^{7}$ platelets were employed after incubation $\left(37^{\circ} \mathrm{C}, 10 \mathrm{~min}\right)$ of platelets with 1.0 unit bovine thrombin and removal of platelets by centrifugation. 
stimulated neutrophils (suspended in cytochalasin $\mathrm{B}, 2.5 \mu \mathrm{g} / \mathrm{ml}$ ) increased in a statistically significant manner $(P<0.02)$, by a factor of $27.6 \%$. Rat neutrophils stimulated with $10^{-6} \mathrm{M}$ fMLP (with cytochalasin B) in the presence of platelets showed nearly a doubling in the $\mathrm{O}_{2}$ response, from 7.41 to $12.4 \mathrm{nmol} \mathrm{O}_{2}$ (Table 1 , Experiment B). In a parallel series of studies, supernatant fluids from thrombinstimulated platelets were able to reproduce the enhancing effects of intact platelets on superoxide responses of fMLP-stimulated neutrophils, resulting in a doubling $(P<.002)$ of the $\mathrm{O} \div$ response (Table 1 , Experiment $\mathrm{C}$ ).

Because platelets are known to secrete substantial quantities of ATP and ADP, and since we have recently shown that superoxide responses of human neutrophils can be enhanced by adenine nucleotides $(1,2)$, the experiments described in Fig. 1 were completed. Rat neutrophils $\left(2 \times 10^{6}\right)$ were exposed to increasing quantities $\left(10^{-7}\right.$ to $\left.10^{-4} M\right)$ of fMLP in the presence or absence of $100 \mu M$ ATP or ADP. Cytochalasin B $(2.5 \mu \mathrm{g} / \mathrm{ml})$ was present in the cell suspensions. The $\mathrm{O}_{2}$ responses were proportional to the concentrations of $\mathrm{MLP}$ and were enhanced by the presence of ATP or ADP, especially at $10^{-5}$ and $10^{-4} M$ concentrations of IMLP. Under these conditions, the superoxide response virtually doubled. Thus, intact platelets, platelet secretion fluids, and ATP or ADP enhance superoxide responses of fMLP-stimulated rat neutrophils.

\section{ATP $\gamma S$ Effects on $O \dot{2}$ in Stimulated Neutrophils}

In order to determine if the enhancing effects of adenine nucleotides on $\mathrm{O} \frac{\dot{2}}{2}$ responses of fMLP-stimulated rat neutrophils also occur with $A T P \gamma S$ (in the presence or absence of cytochalasin B), cells were incubated with $10^{-6} M$ fMLP

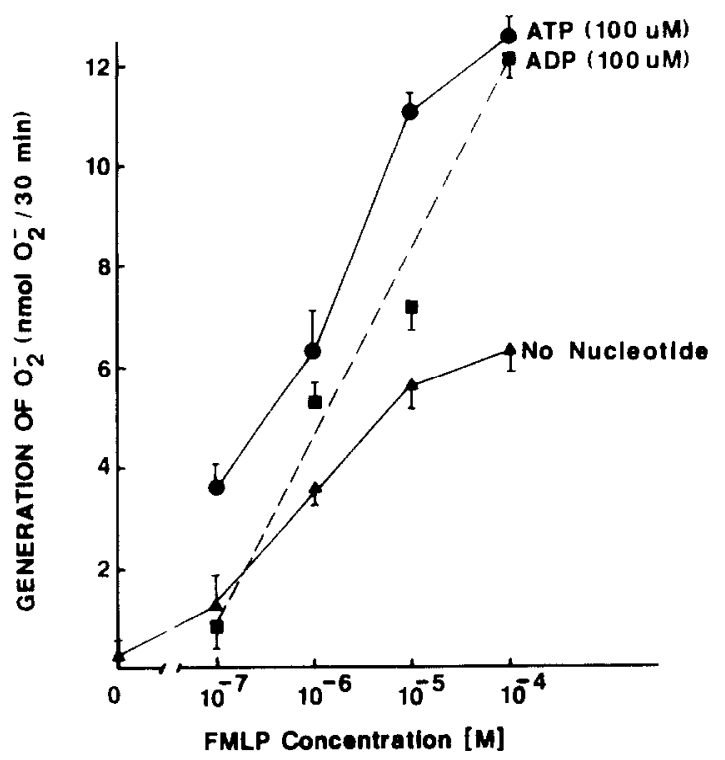

FIG. 1. Ability of ATP and ADP to bring about enhanced $\mathrm{O} \div$ responses in $2 \times 10^{6}$ rat neutrophils with increasing concentrations of $10^{-6} \mathrm{M}$ fMLP in the presence of cytochalasin B $(2.5 \mu \mathrm{g} / \mathrm{ml})$. 
with or without $100 \mu \mathrm{M} A \mathrm{AP} \gamma \mathrm{S}$, in the presence or absence of cytochalasin B (2.5 $\mu \mathrm{g} / \mathrm{ml}$ ). $\mathrm{O} \div \frac{\overline{2}}{2}$ responses are shown in Table 2 and Fig. 2 . Whether or not cytochalasin B was present, the addition of ATP $\gamma \mathrm{S}$ to fMLP-stimulated rat neutrophils resulted in enhanced superoxide responses. The cell concentration was varied according to the presence or absence of cytochalasin $B$ because of the differences in amounts of $\mathrm{O}_{2}^{-}$generated (Table 2). With $5 \times 10^{6}$ neutrophils the addition of ATP $\gamma$ S to $5 \times 10^{6}$ fMLP-stimulated neutrophils in the absence of cytochalasin B resulted in a $30.3 \%$ enhancement $(P<0.03)$ in the $\mathrm{O} \dot{2}$ response. When cytochalasin B was also present, the addition of ATP $\gamma \mathrm{S}$ to a suspension of $2 \times 10^{6}$ fMLP-stimulated neutrophils produced a statistically significant increment (25\% increase, $P<0.001$ ) in the amount of $\mathrm{O} \dot{2}$ generated. The addition of $\mathrm{ATP} \gamma \mathrm{S}$ alone to neutrophils in the presence or absence of cytochalasin $B$ failed to result in an $\mathrm{O} \dot{2}$ response $(<1 \mathrm{nmol})$. Thus, $\mathrm{ATP} \gamma \mathrm{S}$ enhances $\mathrm{O} \dot{2}$ responses of fMLPstimulated neutrophils in the presence or absence of cytochalasin B. Since different numbers of neutrophils were used for the experiments in Table 2, we carried out dose responses of rat neutrophils, varying the cell number from 1 to $5 \times 10^{6}$ in the presence or absence of $2.5 \mathrm{mg}$ cytochalasin $\mathrm{B} / \mathrm{ml}$ and using $10^{-6} M \mathrm{fMLP}$ in the presence or absence of $100 \mu M$ ATP $\gamma S$. The results in Fig. 2 demonstrate that $\mathrm{ATP} \gamma \mathrm{S}$ enhances superoxide responses proportional to the cell number and that the increment in superoxide generation is amplified by the presence of cytochalasin B.

\section{Calcium Transients in Stimulated Neutrophils}

Rat neutrophils were preloaded with the fura 2 probe for measurements of increases in intracellular calcium and were monitored in a fluorescence spectrophotometer in the presence or absence of cytochalasin B $(2.5 \mu \mathrm{g} / \mathrm{ml})$. Cells were incubated with $25 \mu M$ ATP $\gamma S$ or $10^{-6} \mathrm{fMLP}$, or their combination. The reason for selecting the $25 \mu M$ dose for ATP $\gamma$ S was that the $100 \mu M$ concentration of ATP $\gamma$ S produced optical interference in the fluorescence assay (data not shown). The

TABLE 2

Cytochalasin B Effects on Nucleotide Enhancement of $\mathrm{O}_{2}^{-}$Responsfo IN RAT NEUTROPHILS ${ }^{a}$

\begin{tabular}{lcccc}
\hline \multicolumn{1}{c}{ Additions } & Cytochalasin B & Cell number & $\begin{array}{c}\mathrm{O}_{2}^{\circ} \text { Production } \\
\text { (nmol } / 1 / 2 \mathrm{hr})\end{array}$ & Significance \\
\hline & & & $\bar{x} \pm \mathrm{SEM}$ & $P$ Values $^{b}$ \\
fMLP & Absent & $5 \times 10^{6}$ & $8.9+1.6$ & \\
fMLP + ATP $\gamma \mathrm{S}$ & Absent & $5 \times 10^{6}$ & $11.2 \pm 1.7$ & $<0.03$ \\
fMLP & Present & $2 \times 10^{6}$ & $14.9 \pm 1.3$ & \\
fMLP + ATP $\gamma \mathrm{S}$ & Present & $2 \times 10^{6}$ & $18.6 \pm 1.8$ & $<0.001$ \\
ATP $\gamma S$ & Absent & $2 \times 10^{6}$ & $0.37 \pm 0.06$ & \\
ATP $\gamma \mathrm{S}$ & Present & $2 \times 10^{6}$ & $0.29 \pm 0.15$ & NS \\
\hline
\end{tabular}

${ }^{a}$ Experimental conditions: fMLP, $10^{-6} M$; ATP $\gamma S, 100 \mu M$; cytochalasin B, $2.5 \mu \mathrm{g} / \mathrm{ml}$. Data presented are from four separate experiments.

${ }^{b}$ Data in the second line of each pair were compared to those in the first line. 


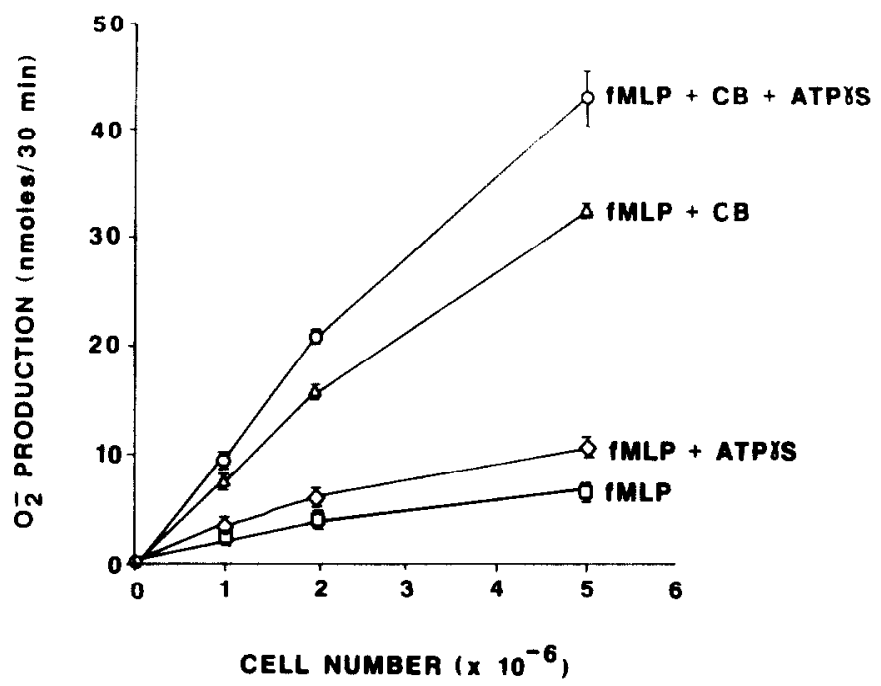

FIG. 2. Generation of $\mathrm{O} \overline{\overline{2}}$ as a function of number of neutrophils incubated with $10^{-6} \mathrm{M} \mathrm{fMLP}$, with or without $100 \mu M$ ATP $\gamma \mathrm{S}$, in the presence or absence of cytochalasin B $(2.5 \mu \mathrm{g} / \mathrm{ml})$.

results with ATP $\gamma$ S in the absence of fMLP are shown in Fig. 3. Brief intracellular calcium transients were produced in rat neutrophils exposed only to $25 \mu M$ ATPyS. In the absence of cytochalasin B (CB), the transients, achieved levels of $334 \mathrm{n} M$ calcium, were of brief duration and had largely disappeared within the first minute. In the presence of cytochalasin B, the transients produced by ATP $\gamma S$ were of greater intensity (achieving peak levels of $527 \mathrm{n} M$ calcium), were of somewhat longer duration, and the intracellular calcium concentrations returned

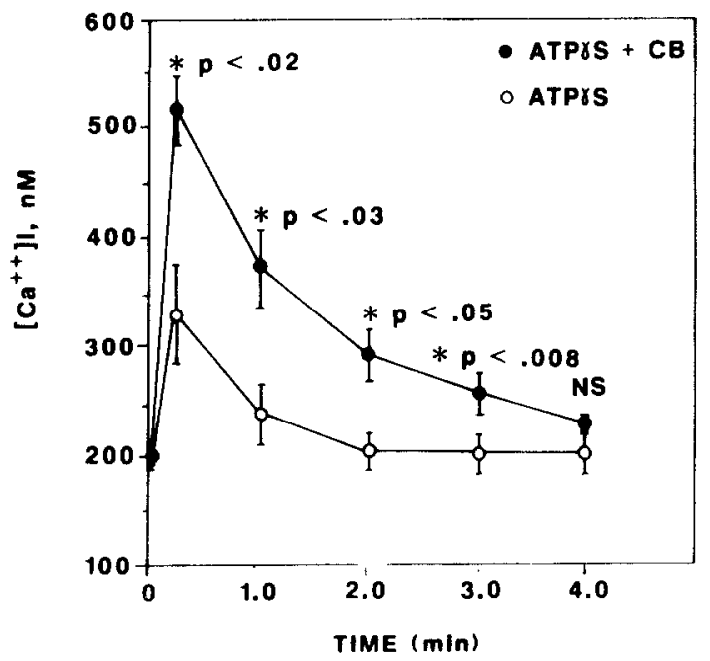

FIG. 3. Intracellular calcium transients in fura 2-loaded neutrophils $\left(5 \times 10^{6}\right)$ incubated with $25 \mu M$ ATP $\gamma \mathrm{S}$ in the presence or absence of cytochalasin B $(2.5 \mu \mathrm{g} / \mathrm{ml})$. For statistical analyses, data at the same intervals of time are compared to cach. 
more slowly to baseline, requiring a period of nearly $4 \mathrm{~min}$ as demonstrated. The calcium concentrations immediately following addition of ATP $\gamma \mathrm{S}$ and at 1.0, 2.0, and $3.0 \mathrm{~min}$ were statistically significantly greater when cytochalasin B was present. It should be stressed that ATP $y S$ (with or without cytochalasin B) failed to induce a measurable $\mathrm{O} \dot{2}$ response in the absence of fMLP (Table 2). When cytochalasin $B$ alone was added to the neutrophil suspension in the absence of any other material, a very small, short-lived and inconsistent calcium transient was noted (data not shown). Thus, as the data in Fig. 3 and Table 2 demonstrate, increases in intracellular calcium caused by ATP $\gamma S$ alone are not sufficient to initiate a superoxide response in the rat neutrophil.

In companion studies calcium transients were measured in fMLP-stimulated rat neutrophils under a variety of conditions (presence or absence of cytochalasin $B$, presence or absence of ATP $\gamma S$ ). The composited results from seven separate experiments are provided in Fig. 4. Statistical evaluation of these data is shown in Table 3. The addition to rat neutrophils of $10^{-6} M$ fMLP in the absence of CB resulted in an immediate intracellular calcium transient (achieving a level of 638 $\mathrm{n} M$ ), which was then followed by a rapid decline in the concentration of intracellular calcium, resulting in a plateau of calcium concentration of approximately $418 \mathrm{nM}$. In the absence of ATP $\gamma \mathrm{S}$, the presence of cytochalasin B did not statistically change the pattern of changes in intracellular calcium with the exception of the 4.0-min interval at which time a statistically significant elevation was found $(P$ $<0.05$ ) in fMLP-stimulated neutrophils (Fig. 4, Table 3). In contrast, a dramatic difference was found in the pattern of intracellular calcium in fMLP-stimulated cells in which cytochalasin B $(2.5 \mu \mathrm{g} / \mathrm{ml})$ and $25 \mu M$ ATP $\gamma \mathrm{S}$ were both present. Under these conditions, the initial early peak of intracellular calcium was the

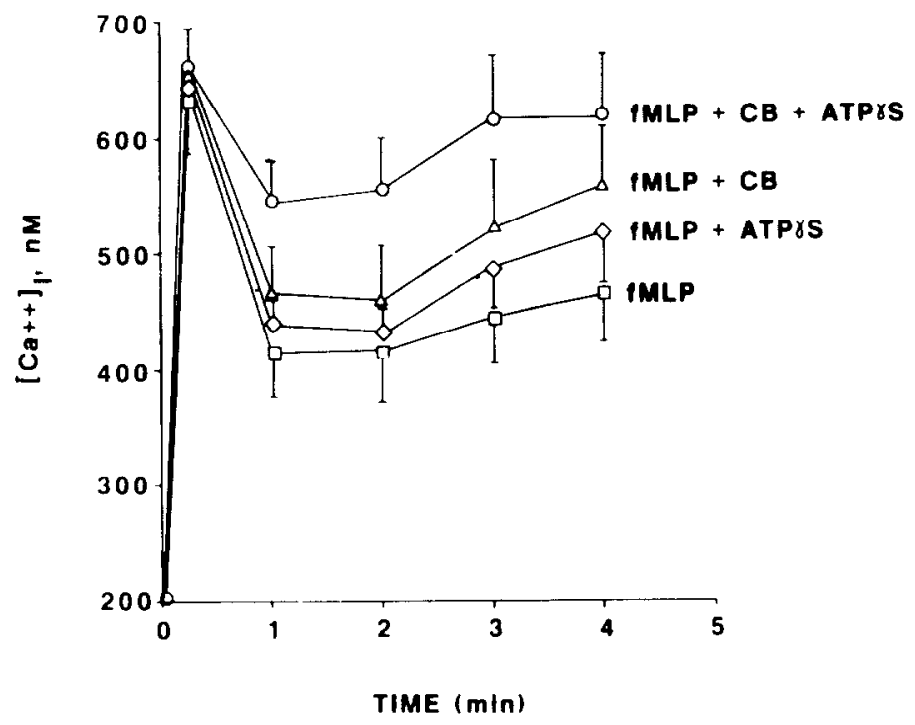

FIG. 4. Intracellular calcium transients in fura 2-loaded neutrophils $\left(5 \times 10^{6}\right)$ incubated with $10^{-6} M$ fMLP with or without $25 \mu M$ ATP $\gamma$, in the presence or absence of cytochalasin B $(2.5 \mu \mathrm{g} / \mathrm{ml})$. 
TABLE 3

Statistical Comparisons of Intracellular Calcium Transients

\begin{tabular}{|c|c|c|c|c|c|c|}
\hline Reference groups" & Rest & 0.25 & 1.0 & 2.0 & 3.0 & 4.0 \\
\hline fMI P vs fMLP + CR & NS & NS & NS & NS & NS & 0.05 \\
\hline fMLP vs fMLP + ATP $\gamma S+$ CB & NS & NS & $<0.0002$ & $<0.0003$ & $<0.0006$ & $<0.0004$ \\
\hline $\mathrm{fMLP}+\mathrm{CB}$ vs fMLP $+\mathrm{ATP} \gamma \mathrm{S}+\mathrm{CB}$ & NS & NS & $<0.04$ & $<0.02$ & $<0.03$ & NS \\
\hline fMLP vs fMLP + ATP $\gamma S$ & NS & NS & NS & NS & NS & NS \\
\hline $\mathrm{fMLP}+\mathrm{ATP} \gamma \mathrm{S}$ vs $\mathrm{fMLP}+\mathrm{ATP} \gamma \mathrm{S}+\mathrm{CB}$ & NS & NS & $<0.005$ & $<0.004$ & $<0.008$ & $<0.01$ \\
\hline
\end{tabular}

"For each reference group, data from seven separate experiments (as shown in Fig. 4) were analyzed.

same ( $P$ value, NS) as that obtained in the absence of ATP $\gamma \mathrm{S}$, but at the intervals of 1.0, 2.0, and 3.0 min the presence of ATP $\gamma$ S in fMLP-stimulated cells caused sustained elevations in the concentration of intracellular calcium, with a plateau being reached between 550 and $625 \mathrm{n} M$ calcium and persisting over most of the 4.0-min interval (Fig. 4). At the intervals of 1.0, 2.0, and $3.0 \mathrm{~min}$, these values were statistically significantly greater $(P$ values of $<0.04,<0.02$, and $<0.03$, respectively) than those obtained in cells similarly treated (fMLP + CB) but in the absence of ATP $\gamma \mathrm{S}$ (Table 3). Finally, in fMLP-stimulated neutrophils in the absence of cytochalasin B, the presence of ATP $\gamma$ S had no statistically significant impact on the intracellular levels of calcium (Fig. 4, Table 3). Thus, statistically significant increases in the levels of intracellular calcium caused by ATP $\gamma \mathrm{S}$ in fMLP-stimulated neutrophils require the presence of cytochalasin B.

\section{Role of Extracellular Calcium in Intracellular Calcium Transients}

Because of the expectation that the plateau in concentration of intracellular calcium achieved between 1-4 min after fMLP stimulation in the presence of cytochalasin B and ATP $\gamma S$ might be related to mobilization of extracellular calcium with resultant influx into the neutrophil of calcium, neutrophils were incubated at various points in time with $25 \mu M$ ATP $\gamma$ S alone or with $10^{-6} M$ fMLP (with or without $25 \mu M \mathrm{ATP} \gamma \mathrm{S}$ ), all in the presence of cytochalasin B. In each experiment measurements of calcium transients were obtained in the presence or absence of $4.0 \mathrm{~m} M$ EGTA (pH 7.4). The results of these experiments are demonstrated in Fig. 5. In frame A, for the calcium transients generated by ATP $\gamma$ S, the addition of EGTA to the cell suspension at the time of addition of ATP $\gamma S$ caused statistically significant reductions in the levels of intracellular calcium at the peak of the calcium transient as well as the 1.0-minute interval, suggesting that extracellular calcium contributes to the intracellular increases in calcium content. In frame B the calcium transients produced by fMLP in the presence of ATP $\gamma$ S were not significantly affected by EGTA during the initial peak of the calcium rise, but at all subsequent intervals $(1-4 \mathrm{~min})$ there were significant reductions in the levels of intracellular calcium when EGTA was present, suggesting that the maintenance of increased levels of intracellular calcium requires availability of extracellular calcium. Finally, in frame $\mathrm{C}$ of Fig. 5, companion experiments were 

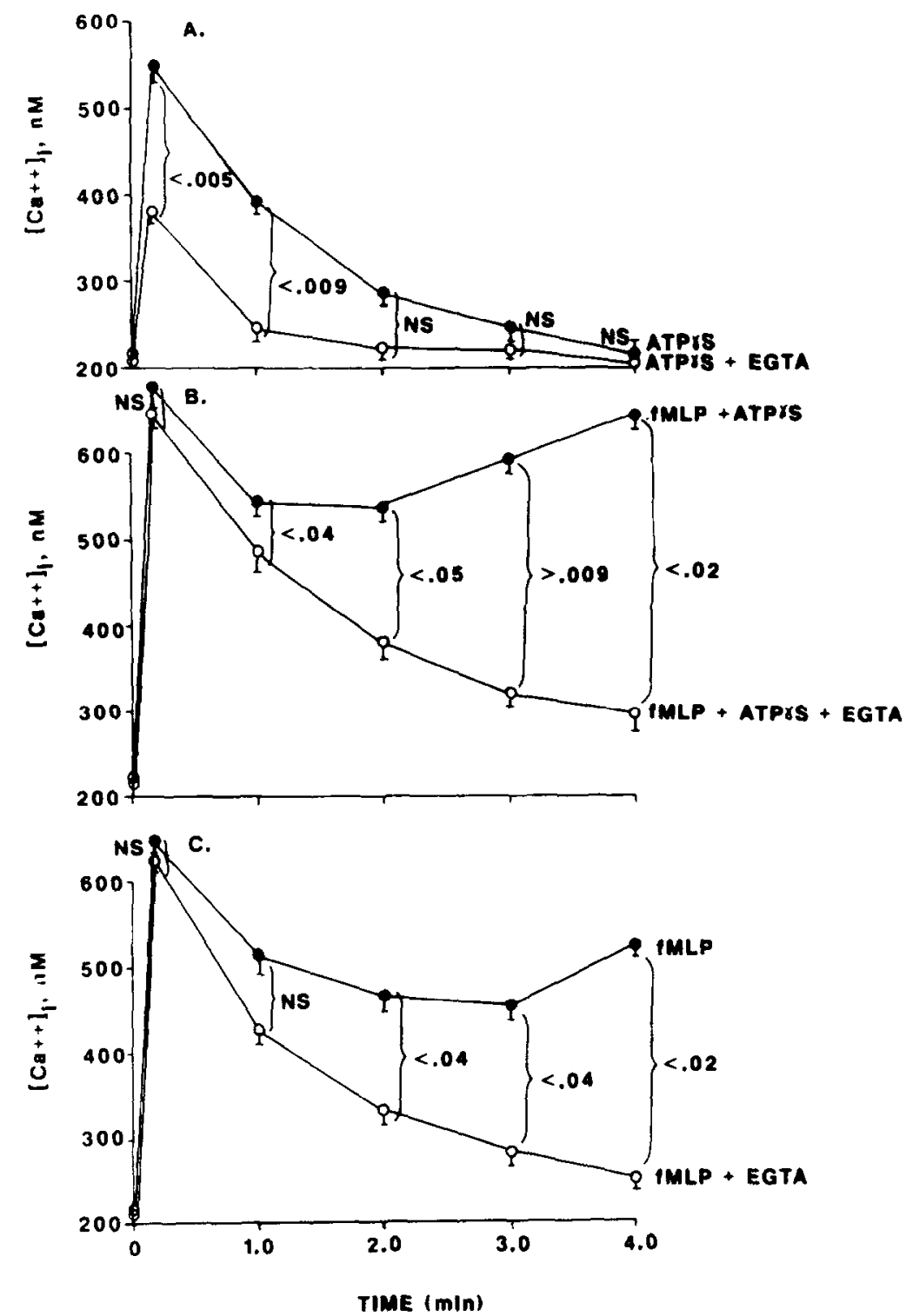

FIG. 5. Requirement of extracellular calcium for intracellular calcium transients in rat neutrophils incubated with $25 \mu M$ ATP $\gamma S$ (A), $10^{-6} M$ fMLP with $25 \mu M$ ATP $\gamma S$ (B), or $10^{-6} M$ fMLP alone (C). In each case a parallel analysis in the presence of $4.0 \mathrm{M}$ EGTA was also carried out. For all experiments cytochalasin $B(2.5 \mu \mathrm{g} / \mathrm{ml})$ was present.

carried out with fMLP in the presence or absence of EGTA. The results were qualitatively rather similar to those also described in frame $B$ : the presence of EGTA in the extracellular fluid did not influence the early peak in the fMLP. induced calcium transient, but the levels of intracellular calcium measured at 2.0, 3.0, and 4.0 minutes were significantly depressed in the presence of EGTA, sug- 
gesting that the late ( $1-4 \mathrm{~min})$ increases in levels of intracellular calcium require mobilization (influx) of extracellular calcium into the neutrophil.

\section{DISCUSSION}

There is good evidence that ATP can modify human and rat neutrophils to cause enhancement of oxygen radical responses $(1,2,5)$ although the mechanism by which this occurs is not currently known. It has recently been suggested that the "priming" effect of ATP on neutrophils is linked to the induction of an intracellular calcium transicnt which is dependent on the availability of extracellular calcium (5). Although we have employed a different methodology (use of EGTA) and have used rat neutrophils, similar results have been obtained, suggesting that a significant portion of the $\mathrm{ATP} \gamma \mathrm{S}$-induced calcium transient requires the mobilization (influx) of extracellular calcium (Fig. 5). This is in contrast to the findings with fMLP in the presence or absence of ATP $\gamma \mathrm{S}$ in which the early peak in the intracellular calcium transient does not appear to require extracellular calcium (Fig. 5). In other studies it has been concluded that, while the intracellular calcium transients generated by fMLP in human neutrophils are chiefly the result of intracellular translocation of calcium, there is also a contribution by extracellular calcium to the increased intracellular levels of calcium (6). What is puzzling is why phorbol ester (PMA) can also prime the neutrophil for enhanced $\mathrm{O}_{2}$ responses even though this does not produce a calcium transient (data not shown). This suggests that either an alternative mechanism in the priming process is brought into play by PMA or that the induction of a calcium transient by ATP or ATP $\gamma \mathrm{S}$ is not directly linked to the priming phenomenon. This question cannot be resolved at present.

There are some suggestions that ATP and ATP $\gamma S$ act at an early step in the signal transduction pathway. Incubation of human neutrophils with ATP results in a limited but measurable level of vitamin $B_{12}$ binding protein but not release of $B$ glucurondase, suggesting that fusion of a select portion of secretory granules to the cell membrane has occurred (7). There is also evidence for increased expression of the CR3 receptor (MO1 antigen) on the surfaces of ATP-treated neutrophils (7). Companion studies in the rat neutrophil have not been completed due to the lack of immunochemical reagents. The lack of $\mathrm{O}_{2}$ production after contact of neutrophils with ATP or ATP $\gamma \mathrm{S}$ suggests that there has not been adequate activation of phospholipase $\mathrm{C}$ to generate amounts of diacylglycerol sufficient for translocation and activation of protein kinase $\mathrm{C}$ and resulting stimulation of NADPH oxidase.

In some respects the priming effects of ATP or ATP $\gamma \mathrm{S}$ are similar to the priming of phagocytic cells by very low concentrations of fMLP (8), platelet-activating factor (9), bacterial lipopolysaccharide (10), and cytokines (IL-1 and tumor necrosis factor) (11). In these situations an $\mathrm{O}_{2}^{-}$response is initialed only after subsequent exposure to the same or another agonist. Rather similar results were reported when neutrophils were exposed to low doses of calcium ionophore A23187 or PMA (12). Cell activation, as defined by enzyme secretion, was not measurable, but subsequent exposure to fMLP caused enhanced enzyme secretory responses. This priming effect required the presence of extracellular calcium 
and was associated with increased binding of fMLP to neutrophils (12). These data are consistent with the hypothesis that the priming phenomenon may, at least in part, be due to fusion of secretory granules with the cell membrane, making the cells hyperresponsive to agonists for which there exist sequestered receptors in secretory granules. It is also possible that the priming phenomenon could be due to fusion to the cell membrane of granule-bound cytochrome B (13) or other essential constituents for the oxidase response, since there is evidence that granules contain an intact $\mathrm{O} \dot{2}$ generating system (14), the transposition of which onto the cell membrane could result in a greater extracellular production of $\mathrm{O} \dot{2}$. These possibilities are topics of current investigation.

\section{ACKNOWLEDGMENT}

These studies were supported in part by NIH Grants HL31963, GM29507, and HL34635.

\section{REFERENCES}

1. Ward P. A., Cunningham, T. W., McCulloch, K. K., Phan, S. H., Powell, J., and Johnson, K. J., Platelet enhancement of $\mathrm{O} \div$ responses in stimulated human neutrophils: Identification of platelet factor as adenine nucleotide. Lab. Invest. 48, 37-47, 1988.

2. Ward, P. A., Cunningham, T. W., McCulloch, K. K., and Johnson, K. J., Regulatory effects of adenosine and adenine nucleotides on oxygen radical responses of neutrophils. Lab. Invest. 58, 438-447, 1988.

3. Ward, P. A., Duque, R. E., Sulavik, M. C., and Johnson, K. J., In vitro and in vivo stimulation of rat neutrophils and alveolar macrophages by immune complexes: Production of $\mathrm{O} \dot{\overline{2}}$ and $\mathrm{H}_{2} \mathrm{O}_{2}$. Amer. J. Pathol. 110, 297, 1983.

4. Grynkiewicz, G., Poenie, M., and Tsien, R. Y., A new generation of $\mathrm{Ca}^{2+}$ indicators with greatly improved fluorescence properties. J. Biol. Chem. 260, 3440-3450, 1985.

5. Kuhns, D. B., Wright, D. G., Nath, J., Kaplan, S. S., and Basford, R. E., ATP induces transients elevations of $\left[\mathrm{Ca}^{2+}\right] \mathrm{i}$ in human neutrophils and primes these cells for enhanced $\mathrm{O} \dot{\overline{2}}$ generation. Lab. Invest. 58, 448-453, 1988.

6. Smolen, J. E., Korchak, A. M., and Wcissmann, G., The roles of extracellular and intracellular calcium in lysosomal enzyme release and superoxide anion generation by human neutrophils. Biochim. Biophys. Acta 667, 512-520, 1981.

7. Freyer, D. R., Boxer, L. A., Axtell, R. A., and Todd, R. F., Stimulation of neutrophil adhesive properties by adenine nucleotides. Blood 70, 205, 1987. [Abstract]

8. McPhail, L. C., Shirdey, P. S., Clayton, C. C., and Snyderman, R., Activation of the respiratory burst enzyme from human neutrophils in a cell free system: Evidence for a soluble factor. J. Clin. Invest. 75, 1735-1739, 1985.

9. Jacob, H. S., and Vercellotti, G. M., Enhancement of granulocyte-mediated endothelial damage by platelet activating factor (PAF). Blood 68, 66A, 1986. [Abstract]

10. Guthrie, L. A., McPhail, L. C., Henson, P. M., and Johnston, R. B., Jr., Priming of neutrophils for enhanced release of oxygen metabolites by bacterial lipopolysaccharide: Evidence for increased activity of the superoxide-producing enzyme. $J$. Exp. Med. 160, 1656-1671, 1984.

11. Warren, J. S., Kunkel, S. L., Cunningham, T. W., Johnson, K. J., and Ward, P. A., Macrophage-derived cytokines amplify immune complex-triggered $\mathrm{O} \dot{\overline{2}}$ responses by rat alveolar macrophages. Amer. J. Pathol. 130, 489-495, 1988.

12. Fletcher, M. P., and Gallin, J. I., Degranulating stimuli increase the availability of receptors in human neutrophils for the chemoattractant f-Met-Leu-Phe. J. Immunol. 124, 1585-1588, 1980.

13. Ohno, Y., Seligmann, B. E., and Gallin, J. I., Cytochrome b translocation to human neutrophil plasma membranes and superoxide release. J. Biol. Chem. 260, 2409-2414, 1985.

14. Borregaard, N., Heiple, J. M., Simmons, E. R., and Clark, R. A., Subcellular localization of the b-cytochrome component of the human neutrophil microbicidal oxidase: Translocation during activation. J. Cell Biol. 97, 52-61, 1983. 